\title{
Thermal and chemical treatment of polymer optical fiber Bragg grating sensors for enhanced mechanical sensitivity
}

\author{
A. Posporia,*, C. A. F. Marques ${ }^{\mathrm{b}}$, D. Sáez-Rodrguez ${ }^{\mathrm{a}}$, K. Nielsen ${ }^{\mathrm{c}}$, \\ O. Bang ${ }^{\mathrm{c}}$, D. J. Webb ${ }^{\mathrm{a}, * *}$ \\ ${ }^{a}$ Aston Institute of Photonic Technologies, Aston University, Aston Triangle, \\ Birmingham, B7 4ET, United Kingdom \\ ${ }^{b}$ Instituto de Telecomunicações and University of Aveiro Physics Department and I3N, \\ Campus de Santiago, 3810-193 Aveiro, Portugal \\ ${ }^{c}$ DTU Fotonik, Department of Photonics Engineering, DK-2800 Kgs. Lyngby, Denmark
}

\begin{abstract}
An investigation of the thermal annealing effects on the strain, stress, and force sensitivities of polymer optical fiber Bragg grating sensors is performed. We demonstrate for the first time that the fiber annealing can enhance both stress and force sensitivities of Bragg grating sensors, with the possible cause being the molecular relaxation of the polymer when fiber is raised above the $\beta$-transition temperature. A simple, cost-effective, but well controlled method for fiber annealing is also presented in this work. In addition, the effects of chemical etching on the strain, stress, and force sensitivities have been investigated. Results show that fiber etching too can increase the force sensitivity, and it can also affect the strain and stress sensitivities of the Bragg grating sensors.
\end{abstract}

Keywords: POF, Bragg grating sensors, annealing, etching, mechanical sensitivity

\footnotetext{
${ }^{*}$ Corresponding author

**Principal corresponding author

Email addresses: a.posporis@aston.ac.uk (A. Pospori), d.j.webb@aston.ac.uk (D. J. Webb)

URL: orcid.org/0000-0003-4866-1361 (A. Pospori), orcid.org/0000-0002-5495-1296 (D. J. Webb)
} 


\section{Introduction}

Polymer optical fiber (POF) sensors have received high attention recently due to their unique properties compared to the conventional silica optical fiber sensors [1].Advantages such as higher flexibility in bending, biocompatibility [2], higher failure strain [3], and higher fracture toughness are significant for many sensing applications. The lower Youngs modulus of POF [4] provides enhanced sensitivity when POF sensors are used for stress [5], pressure [6], and acoustic wave detection [7]. There are also some hydrophilic polymeric materials such as poly(methyl methacrylate) (PMMA) that can absorb water, enabling them to be applied for humidity detection applications [8]. Furthermore, the material properties of polymers can be chemically modified using organic techniques to achieve specific desirable characteristics. An example is the perfluorinated POF, commercially known as CYTOP, in which the carbon-hydrogen bonds have been replaced with carbon-fluorine bonds to reduce the fiber attenuation [9]. One of the drawbacks of POF is its viscoelastic nature. When cyclic loading is applied to the POF sensor, creep and hysteresis effects may be introduced due to the strain and stress phase mismatch [3], which can degrade the accuracy of the sensor reading. It has been demonstrated that these effects can be reduced by applying thermal treatment to POF sensors [10].

Thermal annealing was initially used for POF Bragg grating sensor multiplexing [11], due to the tendence of the anisotropically drawn polymer fiber to physically shrink in length when heated above the $\beta$-transition temperature. The fiber shrinkage can induce a permanent blue shift of the Bragg wavelength, and device multiplexing is feasible by using only one phase mask to record multiple Bragg gratings, with different annealing times. At a later stage, it was demonstrated that fiber annealing can also offer other benefits to POF sensors such as strain [12] and humidity [13] sensitivity enhancement, and a higher operational range for temperature monitoring [14]. Chemical etching has also been utilized to improve the performance of POF Bragg grating sensors. Etching the fiber sensor to reduce its diameter can offer enhanced force sensitivity when force is applied along the fiber axis [15]. This is because for a constant force, the reduction of the area over which force is applied leads to a higher fiber stress. The reduction of the fiber diameter can also improve the response time to humidity changes [16] in POF sensors. This is because the distance between the fiber surface exposed to the external environment and the fiber core is less, which can reduce the time that water 
requires to reach the Bragg gratings location and be detected. However, recently it was shown that etching PMMA with acetone can change not only the physical dimensions of the POF, but also its material properties, such as its Youngs modulus [17].

In this study, both thermal treatment and chemical etching have been applied to POF Bragg grating sensors in order to investigate their influence on strain, stress, and force sensitivity. First, various Bragg grating sensors were photo-inscribed in a single mode PMMA optical fiber using the phase mask technique. Then, each device was characterized with respect to its strain, stress, and force sensitivity. In the next step, some sensors were thermally annealed and some were chemically etched. At this point, the sensitivities of the sensors were characterized again and were compared with the pretreatment sensitivities. Finally, a combination of both treatments was also conducted in order to investigate any possibility for further improvement in performance. Results show that the thermal treatment can enhance not only the strain sensitivity of POF Bragg grating sensors as already reported in the literature, but also the stress and force sensitivities. We also report a simple, cost-effective and well-controlled thermal treatment that has been used to enhance the performance of the POF sensors in this way. In the case where POF etching is used to reduce the fiber diameter for force sensitivity enhancement, changes also occur in the strain and stress sensitivities. However, the changes are not consistent and the possible reasons for this are discussed.

\section{Experimental Details}

\subsection{Fabrication of sensors}

A single mode PMMA based micro-structured optical fiber was used, in which the Bragg grating sensors were photo-inscribed. The fiber core was doped with benzyl dimethyl ketal to enhance the photo-sensitivity of the material in the ultraviolet spectrum region. Details of the fiber and its production are reported in reference [18]. The phase mask technique and a continuous wave He-Cd laser (Kimmon IK3301R-G) operating at $325 \mathrm{~nm}$ were used for the grating inscription as shown in Figure 1. Two phase masks

with periods $\Lambda_{P M}=557.5 \mathrm{~nm}$ and $\Lambda_{P M}=580.0 \mathrm{~nm}$ were used to photo-inscribe gratings with Bragg wavelengths $\lambda_{B}=829 \mathrm{~nm}$ and $\lambda_{B}=862 \mathrm{~nm}$ respectively, indicating that the POF in this wavelength region has an effective refractive 


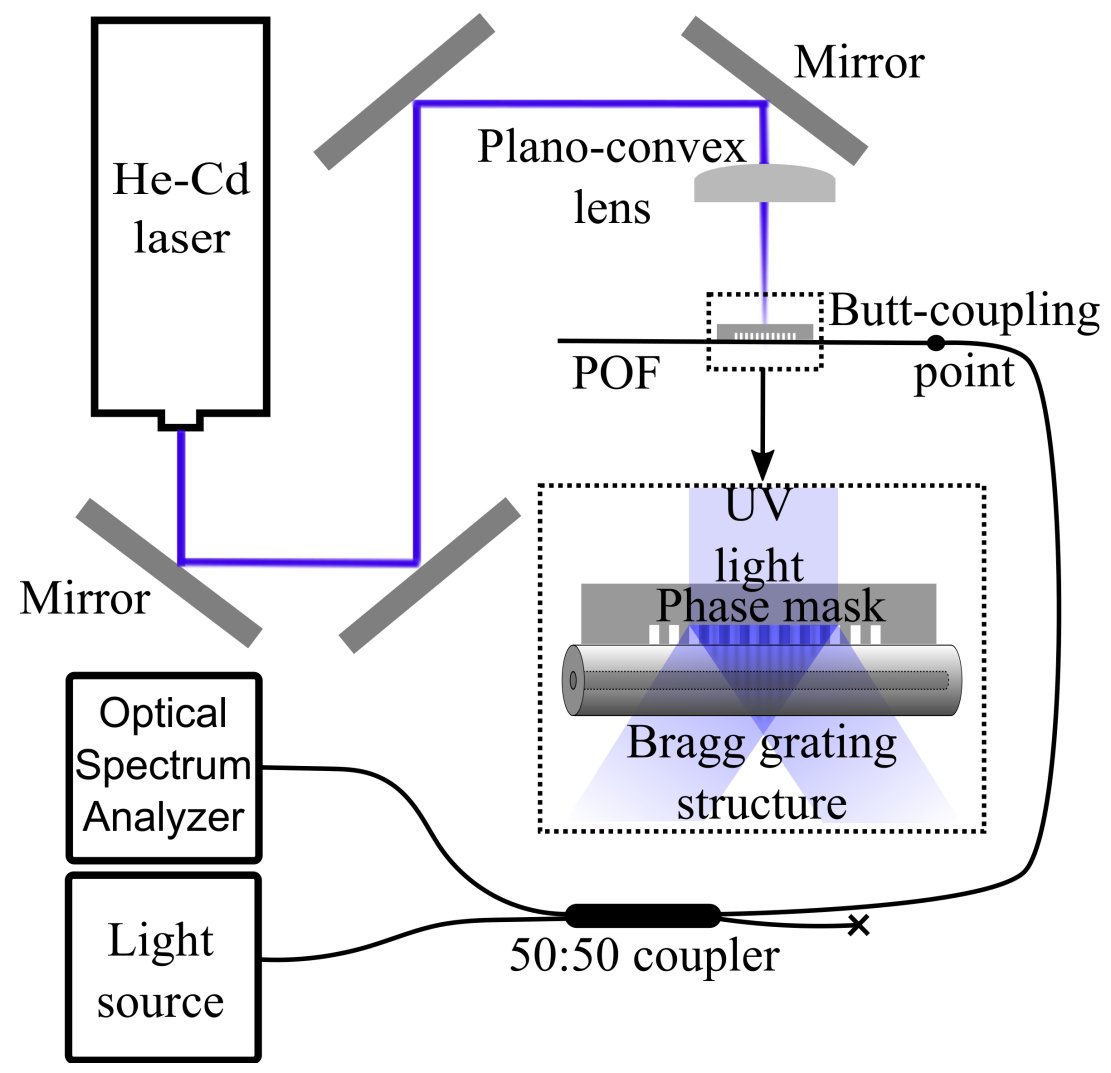

Figure 1: Fabrication setup of POF Bragg grating sensors

index of $n=1.49$ since

$$
\lambda_{B}=n \Lambda_{P M} .
$$

An optical spectrum analyzer (HP 86142A) and a super luminescent diode (Superlum SLD-371) were used to monitor the Bragg grating spectrum in reflection by connecting all together with a 50:50 single mode coupler as shown in Figure 1. The physical length of the inscribed gratings varies between 1.2 and $10 \mathrm{~mm}$, and their total inscription time between 1 and 13 minutes. However, it is believed that these values define only the strength of the Bragg gratings reflectivity and they do not have any impact in the experimental results described in this work. For practical reasons, POF sensors were glued into demountable $\mathrm{FC} / \mathrm{PC}$ connectors after the photo-inscription process, in order to make possible easier interrogation of the Bragg spectrum in contrast with the butt-coupling method. 


\subsection{Characterization of sensors}

The strain, stress, and force sensitivity of each POF Bragg grating sensor was characterized before and after any thermal or chemical treatment was applied. The strain sensitivity can be expressed as $\Delta \lambda_{B} / \varepsilon$, where $\Delta \lambda_{B}$ is the Bragg wavelength shift under the applied fiber strain $\varepsilon$. In order to strain the POF, fiber clamps (Thorlabs HFF01) with V-grooves of $125 \mathrm{~m}$ were used to hold the fiber as shown in Figure 2. However, these fiber clamps were designed for silica optical fibers and as a result the POF was able to slip through the clamp, especially in the case in which it had reduced diameter after etching. As a solution, a polycaprolactone (PCL) material, which has a melting point of $42{ }^{\circ} \mathrm{C}$ [19], was used as a glue to hold the POF in the fiber clamp. First, a small amount of PCL material was melted using a hot plate at $50{ }^{\circ} \mathrm{C}$. Then, the $\mathrm{POF}$ was placed in the $\mathrm{V}$-groove along with some melted PCL material. Finally, the fiber clamping arm was closed with the adjustable knob providing a holding force of $2.0 \mathrm{~N}$. We waited at least 5 minutes before applying any strain to the fiber to allow the PCL material to become solid again and bond with the POF. Using the PCL, we were able to increase the area over which force from the clamp was applied to the fiber and thereby the POF slippage was avoided. After attaching the POF, the translation stage with an accuracy of $1 \mathrm{~m}$ was used to strain the fiber up to $0.5 \%$ along the fiber axis, where POF remains in its elastic limit [20]. After the fiber straining, the POF part that is bonded with the PCL material, was placed on the hot plate at $50{ }^{\circ} \mathrm{C}$. After few seconds the PCL became liquid again and could be removed from the POF.

In order to characterize the stress and force sensitivity, each POF was held perpendicular to the ground and by successively adding masses with a known value, the gravitational force was used to stress the fiber as depicted in Figure 3. The stress equals

$$
\sigma=\frac{F}{A}=\frac{m g}{\pi\left(\frac{d}{2}\right)^{2}},
$$

where $F$ is the gravitational force applied to the cross-sectional area $A$ of the POF. The applied force is equal to the added mass $\mathrm{m}$ multiplied by the gravitational acceleration of the Earth $g=9.8 \mathrm{~m} / \mathrm{s}^{2}$. The cross-sectional area can be calculated when the fiber diameter $d$ is measured with a microscope at the location of the Bragg grating sensor. The fiber diameter increases slightly 


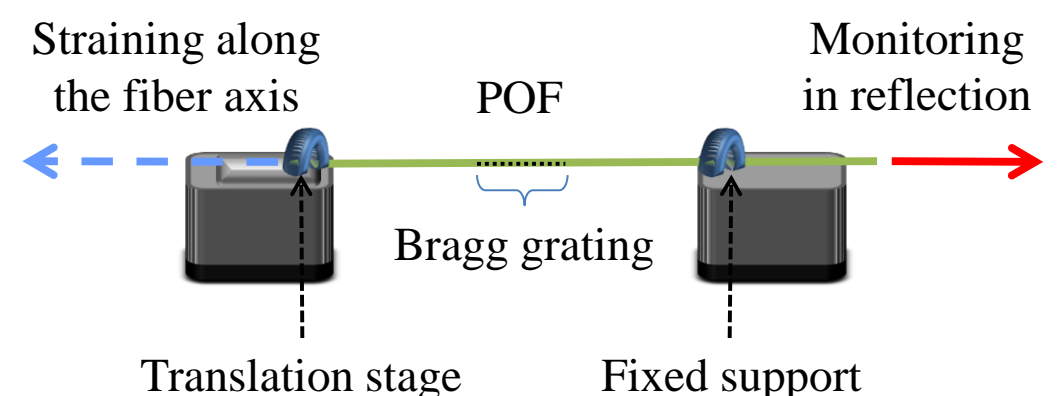

Figure 2: Setup for POF straining

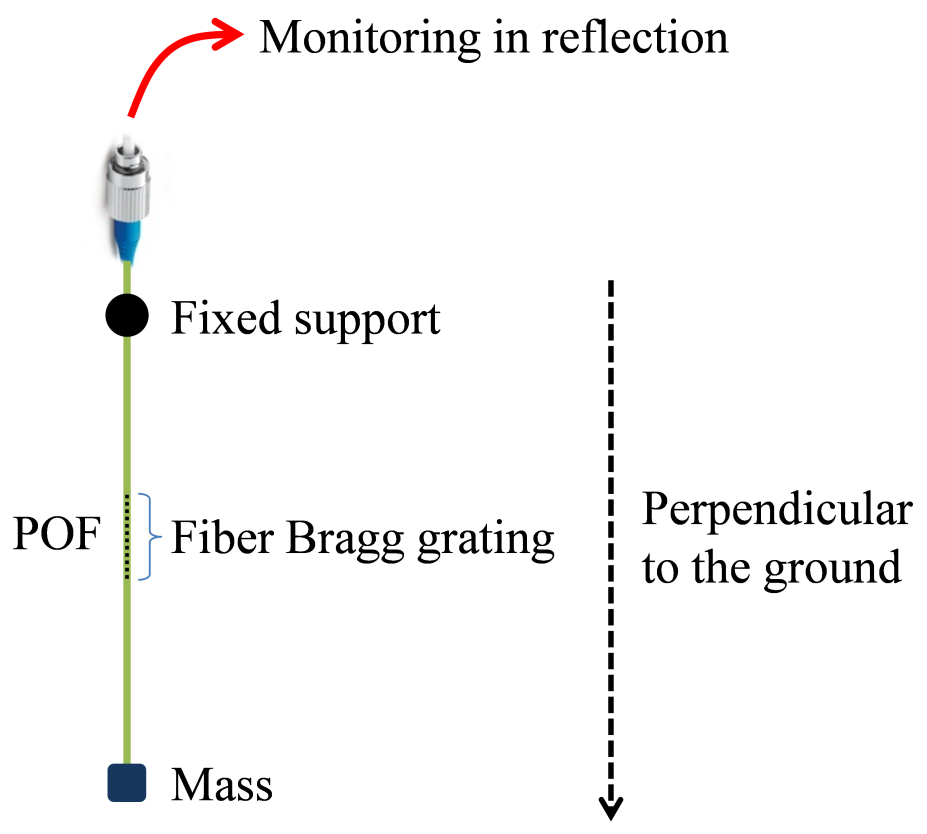

Figure 3: Setup for POF stressing

during the annealing process due to the fiber shrinkage, but it decreases dramatically during the etching process. Therefore, it needs to be measured before and after any treatment process. The stress sensitivity equals $\Delta \lambda_{B} / \sigma$ and the force sensitivity is defined as $\Delta \lambda_{B} / F$. Note that the stress sensitivity is independent of the fiber diameter. We should mention that each time the POF was strained or stressed, a sufficient time (1-3 minutes) was spent 
waiting for the Bragg wavelength to stop shifting due to hysteresis or creep effects, minimizing as much as possible the readout error.

\subsection{Thermal treatment}

In its bulk form, PMMA is mostly amorphous, which means the polymer chains are randomly oriented and entangled with each other. The pulling force used to draw the POF from the preform can stretch the molecule chains along the fiber axis. When the POF is rapidly cooled down to room temperature and solidifies after it is drawn from the oven, the molecular chains become immobilized, preserving a degree of alignment with the fiber axis [21]. The molecular alignment can introduce van der Waals forces between the molecular chains, which are strongly dependent on the molecular orientation and they can influence the thermal and mechanical properties of the polymer [22]. Therefore, the drawing conditions are able to affect the mechanical properties of the POF, such as the Youngs modulus [23, 24]. By introducing enough thermal energy into the polymeric material, the molecular chains become mobilized again [25]. Heating the polymer above the $\beta$-transition temperature in the absence of any external force acting on the polymer material allows each molecule chain to relax towards its equilibrium state, becoming highly amorphous again by reorganizing its conformation $[26,27]$. This molecular rearrangement removes the residual internal stress and as a result the elasticity of the material increases. When annealing the polymer material to achieve molecular stress relaxation, the glass transition temperature should not be exceeded because the molecular movements are not localized anymore and large polymer sections start to move [28, 29], which can erase any gratings recorded in the material. We should also emphasize that the annealing effects or the degree of molecular relaxation of each polymeric material can be different, depending not only on the annealing conditions (annealing duration, temperature, humidity, etc.), but also on their chemical composition, polymerization, degree of cross-linking, and prior thermal history including the fiber drawing conditions [13, 30, 31].

In this study, a thermal treatment is used to relax the stretched molecules of PMMA fiber and investigate the effects of this process on the strain, stress, and force sensitivities of PMMA based Bragg grating sensors. PMMA typically has a $\beta$-transition temperature at $50{ }^{\circ} \mathrm{C}$ [32] and a glass transition temperature at $105{ }^{\circ} \mathrm{C}$ [33]. Previously in the literature when the strain sensitivity was investigated, a comparison was made between different sensors fabricated in annealed and non-annealed fibers [12]. In contrast, in the 
work reported here, the thermal treatment was applied to a sensor after its fabrication and characterization. The reason for choosing this methodology is because of the fluctuation in the drawing conditions, especially in a custom made POF. As is already mentioned above, the drawing conditions can strongly influence the mechanical properties of POF, such as its Youngs modulus $[23,24]$ and any fluctuation of these conditions can lead to non-uniform material properties along the fiber length. This means that the sensors could exhibit different mechanical sensitivity when fabricated at different locations in the same fiber. Characterizing the same sensor before and after any thermal treatment can remove the influence of this factor on the results.

Until now, POF thermal treatment has been only performed by placing the fibers in a climate controlled chamber for extended periods of time from several hours up to several days $[10-14,14,34]$. In this work, a simple, costeffective, but well-controlled thermal annealing was conducted as illustrated in Figure 4. It has already been demonstrated that annealing POF in a high humidity environment can accelerate the annealing process of the material [31]. For this reason, each POF was placed in a container filled with hot water for a short period of time (2 to 30 minutes). The hot water provides a constant 100\% equilibrium relative humidity during the POF annealing, assuring the same thermal and humidity treatment conditions for all sensors. The container is cylindrical with diameter $127 \mathrm{~mm}$ and height $22 \mathrm{~mm}$ and metallic in order to conduct heat from the hot plate as shown in Figure 4. The water temperature was monitored by a mercury thermometer (FISONS THL$290-050 \mathrm{~J})$ and two annealing temperatures $\left(55 \pm 2{ }^{\circ} \mathrm{C}\right.$ or $\left.60 \pm 2{ }^{\circ} \mathrm{C}\right)$ were used for the fiber treatment. After the treatment, each POF sensor was exposed to the ambient room conditions with $40-50 \%$ humidity and $20-25{ }^{\circ} \mathrm{C}$ for at least one hour before its characterization. This is to allow any water that is absorbed during the annealing process to be released out of the POF.

\subsection{Chemical etching}

A Youngs modulus reduction has been reported when a PMMA optical fiber was chemically etched with acetone [17]. The authors claimed that one possible reason for the material property changes could be the molecular chain relaxation due to solvent absorption. It is also highly possible that fiber cladding removal could change the non-uniform stress distribution over the cross-sectional area of the fiber [35]. As is described above, the residual internal stress is created due to the cooling of the stretched molecules during the fiber drawing process. The internal stress distribution could be 


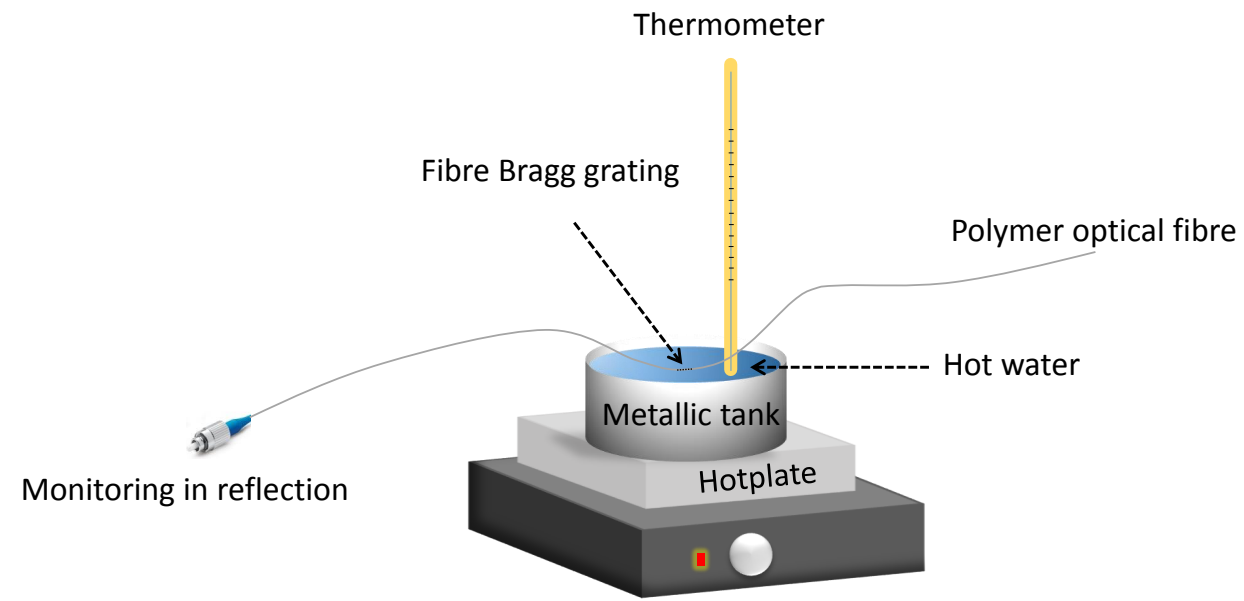

Figure 4: Setup for POF stressing

non-uniform for various reasons, such as fiber designed structure, drawing conditions [36-38], thermal history and cooling rate [39], etc. For example, let us assume a homogeneous fiber drawing, where all the molecules are stretched when they are heated up well above their glass transition temperature. While continuing the drawing process, eventually the fiber starts to cool down and the molecules located on the outer layer of POF are cooled faster than the molecules located closer to the fiber core. At this stage, there is a possibility for the inner molecules to relax to a greater degree before they become immobile, as a result the residual stress may be higher towards the side of the fibre [37]. Therefore, by etching the fiber that has a non-uniform stress distribution along its cross-section, the average internal stress could be changed.

In this study, POF etching is used to investigate if there are any changes on the strain and stress sensitivities of POF Bragg grating sensors. The force sensitivity is naturally expected to increase with etching due to the reduction of the cross-sectional area of the fiber. In order to etch the PMMA optical fibers, they have been placed in a container filled with pure acetone for 4-7 minutes and the fiber diameters were reduced by $14-48 \%$. Then, the sensors have been characterized again with the same method described above. 


\section{Results}

Table 1 lists the annealing duration and annealing temperature applied to 9 different sensors and Table 2 lists their strain, stress, and force sensitivities before and after the annealing. The results show that in all cases the thermal treatment enhanced not only the strain, but also the stress and force sensitivities of the POF Bragg grating sensors. The difference in annealing duration and annealing temperature do not clearly lead to any consistent difference in these results and further investigation of the influence of those parameters is out of the scope of this study. The only noticeable difference is on the

Table 1: Annealing duration and temperature for sensors 1-9

\begin{tabular}{ccc}
\hline Sensor & $\begin{array}{c}\text { Annealing } \\
\text { Duration } \\
(\mathbf{m i n})\end{array}$ & $\begin{array}{c}\text { Annealing } \\
\text { Temperature } \\
\left({ }^{\circ} \mathbf{C}\right) \pm \mathbf{2}\end{array}$ \\
\hline 1 & 2 & 60 \\
2 & 4 & 60 \\
3 & 4 & 60 \\
4 & 30 & 55 \\
5 & 30 & 55 \\
6 & 30 & 55 \\
7 & 30 & 55 \\
8 & 30 & 60 \\
9 & 30 & 60 \\
\hline \hline
\end{tabular}

fiber shrinkage and the Bragg wavelength shift. Figure 5 shows the Bragg wavelength shift due to the POF thermal treatment in water at $55 \pm 2{ }^{\circ} \mathrm{C}$ and $60 \pm 2{ }^{\circ} \mathrm{C}$. The results do not show the initial temperature related blue wavelength shift that occurs as the sensor is submerged in the hot water. The fitting equations show the complex heterogeneous nature of molecular relaxation and fiber shrinkage. It is demonstrated that the POF shrinkage follows a dynamic exponential decay [31], which depends on the annealing conditions and the POF history. In general, as the annealing temperature and humidity increases, the rate of fiber annealing is higher. This is the reason for having second order exponential decay when annealing the fiber at $60 \pm 2{ }^{\circ} \mathrm{C}$ (Figure 5). Using even higher annealing temperatures, the Bragg wavelength shift is expected to follow more stretched exponential decay, as has already been reported in reference [31]. 
Table 2: Strain, stress, and force sensitivities before and after the thermal treatment

\begin{tabular}{ccccccc}
\hline \multirow{2}{*}{ Sensor } & \multicolumn{2}{c}{$\begin{array}{c}\text { Strain } \\
\text { Sensitivity } \\
(\mathbf{p m} / \boldsymbol{\mu \varepsilon})\end{array}$} & \multicolumn{2}{c}{$\begin{array}{c}\text { Stress } \\
\text { Sensitivity } \\
(\mathbf{p m} / \mathbf{k P a})\end{array}$} & \multicolumn{2}{c}{$\begin{array}{c}\text { Force } \\
\text { Sensitivity } \\
(\mathbf{p m} / \mathbf{m N})\end{array}$} \\
\cline { 2 - 7 } & Before & After & Before & After & Before & After \\
\hline \multirow{2}{*}{1} & 0.681 & 0.739 & 0.141 & 0.217 & 10.92 & 13.65 \\
& \pm 0.009 & \pm 0.019 & \pm 0.018 & \pm 0.022 & \pm 1.37 & \pm 1.37 \\
\hline \multirow{2}{*}{2} & 0.708 & 0.902 & 0.180 & 0.260 & 10.92 & 14.33 \\
& \pm 0.007 & \pm 0.016 & \pm 0.023 & \pm 0.025 & \pm 1.37 & \pm 1.37 \\
\hline \multirow{2}{*}{3} & 0.541 & 0.694 & 0.147 & 0.201 & 10.92 & 13.65 \\
& \pm 0.019 & \pm 0.012 & \pm 0.019 & \pm 0.021 & \pm 1.37 & \pm 1.37 \\
\hline \multirow{2}{*}{4} & 0.711 & 0.879 & 0.163 & 0.191 & 12.24 & 14.37 \\
& \pm 0.007 & \pm 0.020 & \pm 0.026 & \pm 0.018 & \pm 1.96 & \pm 1.34 \\
\hline \multirow{2}{*}{5} & 0.726 & 0.875 & 0.176 & 0.208 & 11.44 & 13.26 \\
& \pm 0.007 & \pm 0.020 & \pm 0.009 & \pm 0.011 & \pm 0.59 & \pm 0.62 \\
\hline \multirow{2}{*}{6} & 0.728 & 0.889 & 0.182 & 0.220 & 11.87 & 14.10 \\
& \pm 0.004 & \pm 0.033 & \pm 0.006 & \pm 0.011 & \pm 0.39 & \pm 0.62 \\
\hline \multirow{2}{*}{7} & 0.690 & 0.883 & 0.191 & 0.221 & 12.00 & 13.87 \\
& \pm 0.011 & \pm 0.011 & \pm 0.016 & \pm 0.020 & \pm 0.98 & \pm 1.23 \\
\hline \multirow{2}{*}{8} & 0.727 & 0.849 & 0.197 & 0.258 & 13.56 & 17.88 \\
& \pm 0.011 & \pm 0.011 & \pm 0.023 & \pm 0.023 & \pm 1.54 & \pm 1.54 \\
\hline \multirow{2}{*}{9} & 0.771 & 0.943 & 0.173 & 0.202 & 13.41 & 14.64 \\
& \pm 0.011 & \pm 0.011 & \pm 0.024 & \pm 0.026 & \pm 1.85 & \pm 1.85 \\
\hline \hline
\end{tabular}

After the thermal treatment, sensors 7-9 were placed in pure acetone solvent for 7 minutes to investigate the etching effects on strain, stress, and force sensitivities. Table 3 shows the diameter reduction due to etching. The diameter reduction depends mostly on the duration of etching, though there is some variation, possibly due to contamination of the acetone solvent, which was used for several sensors. Table 4 lists the strain, stress, and force sensitivities of the annealed sensors 7-9 before and after the chemical etching. Strain sensitivity seems to be slightly reduced and the stress sensitivity increases. Understandably, the force sensitivities have been dramatically increased mostly due to the diameter reduction of the fibers. In order to further investigate the chemical etching effects on POF sensors, an additional 9 sensors were characterized before and after etching without applying 


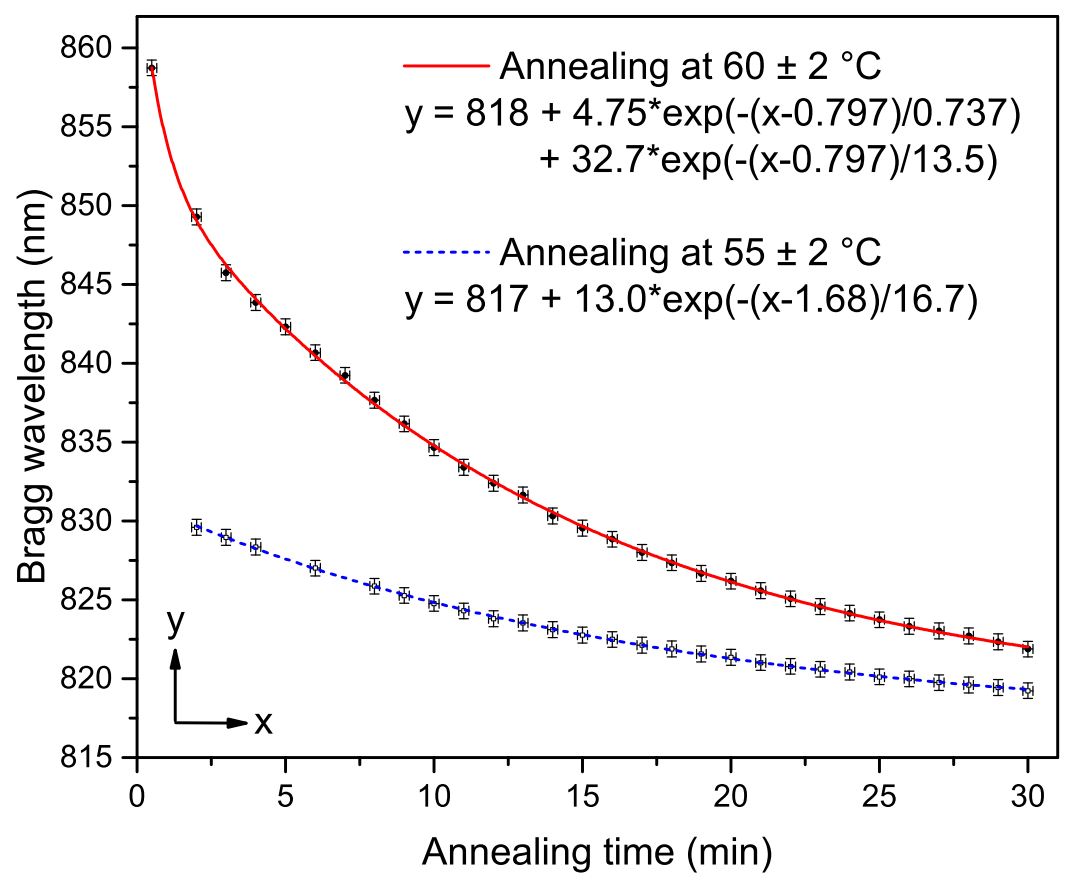

Figure 5: POF annealing at $55 \pm 2{ }^{\circ} \mathrm{C}$ and $60 \pm 2{ }^{\circ} \mathrm{C}$

Table 3: Etching duration and diameter reduction of sensors 7-9

\begin{tabular}{ccccc}
\hline Sensor & $\begin{array}{c}\text { Etching } \\
\text { Duration } \\
(\boldsymbol{m i n})\end{array}$ & $\begin{array}{c}\text { Diameter } \\
\text { before } \\
\text { etching }(\boldsymbol{\mu m})\end{array}$ & $\begin{array}{c}\text { Diameter } \\
\text { after } \\
\text { etching }(\boldsymbol{\mu m})\end{array}$ & $\begin{array}{c}\text { Diameter } \\
\text { reduction } \\
\boldsymbol{\%}\end{array}$ \\
\hline 7 & 7 & 143 & 98 & 31 \\
8 & 7 & 136 & 92 & 32 \\
9 & 7 & 133 & 69 & 48 \\
\hline \hline
\end{tabular}

any thermal treatment process (annealing). Table 5 shows the diameter reduction of the sensors for various etching durations. The effects of chemical etching on these POF sensors are listed in Table 6. The results from this more comprehensive study show inconsistent data regarding the strain and stress sensitivity changes after etching, and therefore a further analysis has been conducted. Figure 6 shows the percentage growth of strain sensitivity of each sensor after etching and the average mean value is only $3.4 \% \pm 4.7 \%$, comparable to the error associated with the measurements. The average percentage growth of stress sensitivity after etching is $11.6 \% \pm 7.6 \%$ as depicted 
Table 4: Strain, stress, and force sensitivities before and after etching the annealed sensors

\begin{tabular}{ccccccc}
\hline \multirow{2}{*}{ Sensor } & \multicolumn{2}{c}{$\begin{array}{c}\text { Strain } \\
\text { Sensitivity } \\
(\mathbf{p m} / \boldsymbol{\mu \varepsilon})\end{array}$} & \multicolumn{2}{c}{$\begin{array}{c}\text { Stress } \\
\text { Sensitivity } \\
(\mathbf{p m} / \mathbf{k P a})\end{array}$} & \multicolumn{2}{c}{$\begin{array}{c}\text { Force } \\
\text { Sensitivity } \\
(\mathbf{p m} / \mathbf{m N})\end{array}$} \\
\cline { 2 - 7 } & Before & After & Before & After & Before & After \\
\hline \multirow{2}{*}{7} & 0.883 & 0.854 & 0.221 & 0.259 & 13.87 & 34.05 \\
& \pm 0.011 & \pm 0.022 & \pm 0.020 & \pm 0.020 & \pm 1.23 & \pm 2.63 \\
\hline \multirow{2}{*}{8} & 0.849 & 0.755 & 0.258 & 0.260 & 17.88 & 39.53 \\
& \pm 0.011 & \pm 0.012 & \pm 0.023 & \pm 0.011 & \pm 1.54 & \pm 1.60 \\
\hline \multirow{2}{*}{9} & 0.943 & 0.848 & 0.202 & 0.241 & 14.64 & 64.97 \\
& \pm 0.011 & \pm 0.012 & \pm 0.026 & \pm 0.006 & \pm 1.85 & \pm 1.75 \\
\hline \hline
\end{tabular}

Table 5: Etching duration and diameter reduction of sensors 10-18

\begin{tabular}{ccccc}
\hline Sensor & $\begin{array}{c}\text { Etching } \\
\text { Duration } \\
(\mathbf{m i n})\end{array}$ & $\begin{array}{c}\text { Diameter } \\
\text { before } \\
\text { etching }(\boldsymbol{\mu m})\end{array}$ & $\begin{array}{c}\text { Diameter } \\
\text { after } \\
\text { etching }(\boldsymbol{\mu m})\end{array}$ & $\begin{array}{c}\text { Diameter } \\
\text { reduction } \\
\%\end{array}$ \\
\hline 10 & 4 & 144 & 123 & 14 \\
11 & 4.5 & 156 & 116 & 26 \\
12 & 5 & 152 & 97 & 37 \\
13 & 5 & 139 & 92 & 34 \\
14 & 5.5 & 129 & 81 & 37 \\
15 & 7 & 130 & 72 & 44 \\
16 & 7 & 152 & 91 & 41 \\
17 & 7 & 142 & 84 & 41 \\
18 & 7 & 131 & 84 & 36 \\
\hline \hline
\end{tabular}

in Figure 7. This means that we cannot conclusively state whether etching can improve the strain or stress sensitivity, but in any case any such improvement will be very small. The force sensitivity is increased dramatically in all cases, due to the reduction in cross-sectional area. The etched sensors 16-18 were then annealed at $55 \pm 2{ }^{\circ} \mathrm{C}$ for 30 minutes. Table 7 shows that by etching and then annealing the sensors, their strain, stress, and force sensitivities can be all dramatically enhanced. In this case, most of the improvement in strain and stress sensitivity comes from the annealing process, while the etching can further enhance the force sensitivity of the sensor. 
Table 6: Strain, stress, and force sensitivities before and after the chemical etching

\begin{tabular}{ccccccc}
\hline \multirow{2}{*}{ Sensor } & \multicolumn{2}{c}{$\begin{array}{c}\text { Strain } \\
\text { Sensitivity } \\
(\mathbf{p m} / \boldsymbol{\mu \varepsilon})\end{array}$} & \multicolumn{2}{c}{$\begin{array}{c}\text { Stress } \\
\text { Sensitivity } \\
(\mathbf{p m} / \mathbf{k P a})\end{array}$} & \multicolumn{2}{c}{$\begin{array}{c}\text { Force } \\
\text { Sensitivity } \\
(\mathbf{p m} / \mathbf{m N})\end{array}$} \\
\cline { 2 - 7 } & Before & After & Before & After & Before & After \\
\hline \multirow{2}{*}{10} & 0.788 & 0.791 & 0.194 & 0.214 & 11.98 & 17.95 \\
& \pm 0.013 & \pm 0.008 & \pm 0.009 & \pm 0.003 & \pm 0.53 & \pm 0.25 \\
\hline \multirow{2}{*}{11} & 0.717 & 0.746 & 0.209 & 0.202 & 10.88 & 19.12 \\
& \pm 0.016 & \pm 0.008 & \pm 0.003 & \pm 0.013 & \pm 0.13 & \pm 1.19 \\
\hline \multirow{2}{*}{12} & 0.720 & 0.667 & 0.194 & 0.238 & 10.62 & 32.34 \\
& \pm 0.040 & \pm 0.026 & \pm 0.004 & \pm 0.002 & \pm 0.23 & \pm 0.23 \\
\hline \multirow{2}{*}{13} & 0.726 & 0.768 & 0.185 & 0.200 & 12.28 & 30.07 \\
& \pm 0.040 & \pm 0.034 & \pm 0.007 & \pm 0.010 & \pm 0.46 & \pm 1.46 \\
\hline \multirow{2}{*}{14} & 0.728 & 0.810 & 0.189 & 0.217 & 14.54 & 42.06 \\
& \pm 0.040 & \pm 0.027 & \pm 0.003 & \pm 0.005 & \pm 0.23 & \pm 0.91 \\
\hline \multirow{2}{*}{15} & 0.810 & 0.860 & 0.195 & 0.201 & 14.72 & 49.23 \\
& \pm 0.019 & \pm 0.010 & \pm 0.002 & \pm 0.002 & \pm 0.13 & \pm 0.39 \\
\hline \multirow{2}{*}{16} & 0.709 & - & 0.199 & 0.191 & 10.94 & 29.59 \\
& \pm 0.020 & - & \pm 0.028 & \pm 0.012 & \pm 1.54 & \pm 1.54 \\
\hline \multirow{2}{*}{17} & 0.649 & & 0.169 & 0.183 & 10.70 & 33.45 \\
& \pm 0.010 & - & \pm 0.009 & \pm 0.011 & \pm 0.56 & \pm 1.54 \\
\hline \multirow{2}{*}{18} & 0.657 & & 0.146 & 0.211 & 10.82 & 37.76 \\
& \pm 0.010 & - & \pm 0.017 & \pm 0.010 & \pm 1.24 & \pm 1.23 \\
\hline \hline \multirow{2}{*}{15} & & & & & &
\end{tabular}

Table 7: Strain, stress, and force sensitivities before and after both treatments

\begin{tabular}{ccccccc}
\hline \multirow{2}{*}{ Sensor } & \multicolumn{2}{c}{$\begin{array}{c}\text { Strain } \\
\text { Sensitivity } \\
(\mathbf{p m} / \boldsymbol{\mu \varepsilon})\end{array}$} & \multicolumn{2}{c}{$\begin{array}{c}\text { Stress } \\
\text { Sensitivity } \\
(\mathbf{p m} / \mathbf{k P a})\end{array}$} & \multicolumn{2}{c}{$\begin{array}{c}\text { Force } \\
\text { Sensitivity } \\
(\mathbf{p m} / \mathbf{m N})\end{array}$} \\
\cline { 2 - 7 } & Before & After & Before & After & Before & After \\
\hline \multirow{2}{*}{16} & 0.709 & 0.834 & 0.199 & 0.232 & 10.94 & 32.17 \\
& \pm 0.020 & \pm 0.022 & \pm 0.028 & \pm 0.017 & \pm 1.54 & \pm 2.15 \\
\hline \multirow{2}{*}{17} & 0.649 & 0.944 & 0.169 & 0.200 & 10.70 & 36.63 \\
& \pm 0.010 & \pm 0.014 & \pm 0.009 & \pm 0.011 & \pm 0.56 & \pm 1.54 \\
\hline \multirow{2}{*}{18} & 0.657 & 0.962 & 0.146 & 0.223 & 10.82 & 37.14 \\
& \pm 0.010 & \pm 0.014 & \pm 0.017 & \pm 0.001 & \pm 1.24 & \pm 0.19 \\
\hline \hline
\end{tabular}




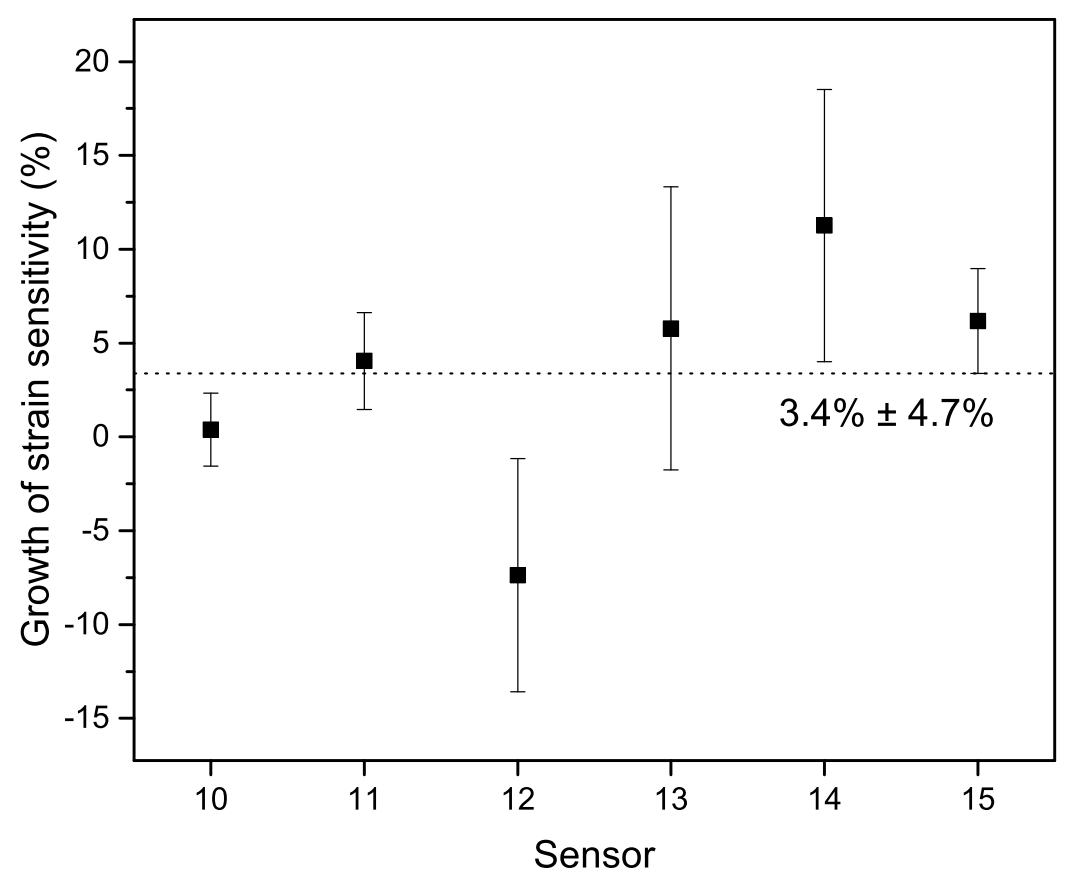

Figure 6: Average growth of strain sensitivity after etching

\section{Conclusion}

In this study, the thermal annealing and the chemical etching effects on strain, stress, and force sensitivities of POF Bragg grating sensors have been investigated. A simple, cost-effective, but well controlled thermal treatment is presented for annealing the POF sensors. Previously, it was demonstrated that strain sensitivity can be enhanced by fiber annealing. In this work, we demonstrate for the first time that stress and force sensitivities can be also enhanced. The reason for the enhancement is probably the molecular relaxation of the material, when the $\mathrm{POF}$ is exposed above the $\beta$-transition temperature. Chemical etching the POF Bragg grating sensors also affects the strain and stress sensitivities. A hypothetical reason could be the nonuniform internal stress distribution in the fiber, which changes when some of the fiber cladding is etched. In general, chemical etching is preferable when the POF Bragg grating sensors are used in force detection applications. On the other hand, the thermal treatment can improve the performance of the sensors in both strain and stress sensing applications. A combination of both annealing and etching may be beneficial depending on the application 


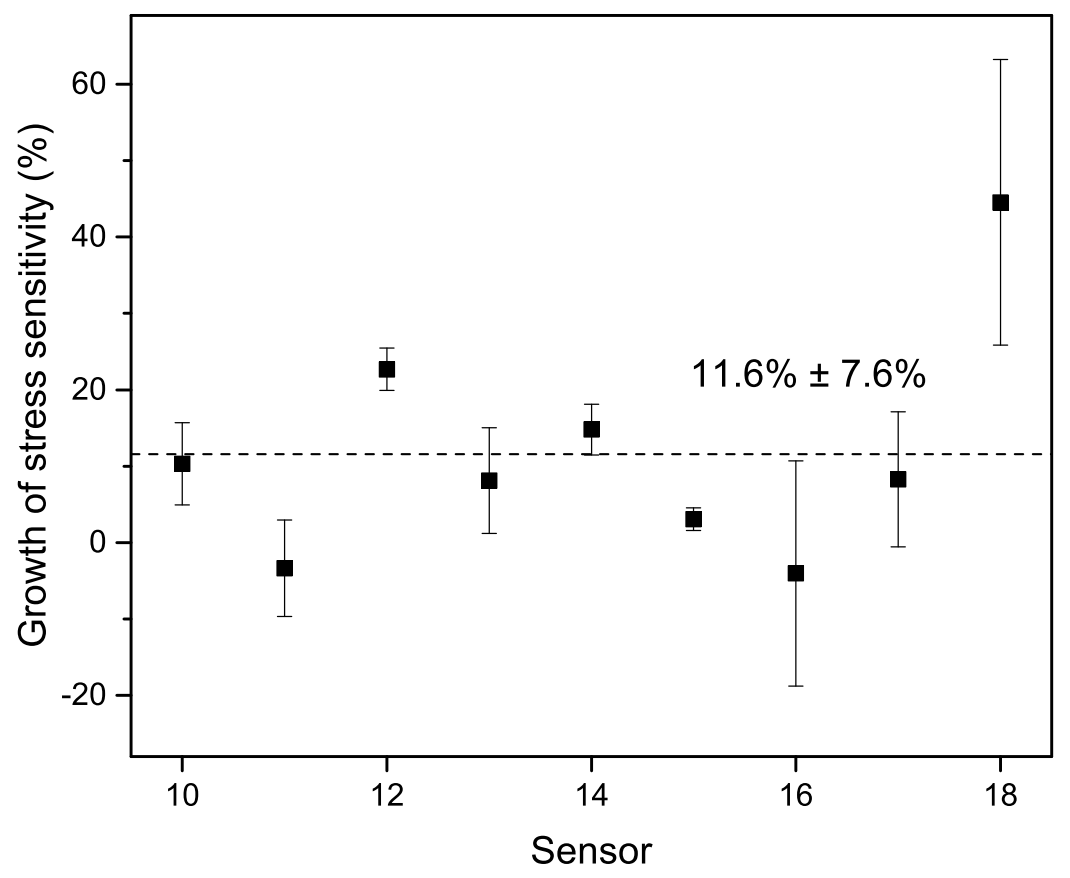

Figure 7: Average growth of stress sensitivity after etching

type. One example could be for humidity detection applications; the thermal treatment allows the molecules to become amorphous again, providing the ability to absorb water more easily than the aligned molecules. In addition, etching the fiber to reduce its diameter reduces the time the water requires to reach the Bragg grating sensor in POF core. Therefore, a combination of both annealing and etching could be beneficial for such applications.

\section{Acknowledgements}

The research leading to these results has received funding from the People Programme (Marie Curie Actions) of the European Union's Seventh Framework Programme FP7/2007-2013/ under REA grant agreement No. 608382. This work was supported by Marie Curie Intra European Fellowships included in the 7th Framework Program of the European Union (projects PIEF-GA-2013-628604 and PIEF-GA-2011-302919). The financial support from FCT through the fellowship SFRH/BPD/109458/2015 is also acknowledged. 


\section{References}

[1] D. J. Webb, Fibre Bragg grating sensors in polymer optical fibres, Measurement Science and Technology 26 (9) (2015) 092004.

[2] F. Bischoff, Organic polymer biocompatibility and toxicology, Clinical Chemistry 18 (9) (1972) 869-94.

[3] M. C. J. Large, J. H. Moran, L. Ye, The role of viscoelastic properties in strain testing using microstructured polymer optical fibres (mPOF), Measurement Science and Technology 20 (3) (2009) 034014.

[4] M. Silva-Lopez, A. Fender, W. N. Macpherson, J. S. Barton, J. D. C. Jones, Strain and temperature sensitivity of a single-mode polymer optical fiber, Optics Letters 30 (2005) 3129-3131.

[5] T. X. Wang, Y. H. Luo, G. D. Peng, Q. J. Zhang, High-sensitivity stress sensor based on Bragg grating in BDK-doped photosensitive polymer optical fiber, in: J. Canning, G. D. Peng (Eds.), Third Asia Pacific Optical Sensors Conference, Vol. 8351 of Proc. SPIE 8351, 2012, pp. 83510M-83510M.

[6] C. A. F. Marques, A. Pospori, D. Saez-Rodriguez, K. Nielsen, O. Bang, D. J. Webb, Aviation Fuel Gauging Sensor Utilizing Multiple Diaphragm Sensors Incorporating Polymer Optical Fiber Bragg Gratings, IEEE Sensors Journal 16 (15) (2016) 6122-6129.

[7] C. Broadway, D. Gallego, A. Pospori, M. Zubel, D. J. Webb, K. Sugden, G. Carpintero, H. Lamela, Microstructured polymer optical fibre sensors for opto-acoustic endoscopy, in: Proc. SPIE, Vol. 9886, 2016, pp. 98860S-98860S-6.

[8] W. Zhang, D. J. Webb, G. D. Peng, Investigation Into Time Response of Polymer Fiber Bragg Grating Based Humidity Sensors, Journal of Lightwave Technology 30 (8) (2012) 1090-1096.

[9] S. Ando, T. Matsuura, S. Sasaki, Perfluorinated polymers for optical waveguides, Chemtech 24 (12) (1994) 20-27.

[10] A. Abang, D. J. Webb, Effects of annealing, pre-tension and mounting on the hysteresis of polymer strain sensors, Measurement Science and Technology 25 (1) (2014) 015102. 
[11] I. P. Johnson, D. J. Webb, K. Kalli, Utilisation of thermal annealing to record multiplexed FBG sensors in multimode microstructured polymer optical fibre, in: W. J. Bock, J. Albert, X. Bao (Eds.), 21st International Conference on Optical Fiber Sensors (OFS21), Vol. 7753, Proc. SPIE, 2011, pp. 7327-7753.

[12] W. Yuan, A. Stefani, M. Bache, T. Jacobsen, B. Rose, N. HerholdtRasmussen, F. K. Nielsen, S. Andresen, O. B. Sorensen, K. S. Hansen, O. Bang, Improved thermal and strain performance of annealed polymer optical fiber Bragg gratings, Optics Communications 284 (1) (2011) 176182.

[13] G. Woyessa, K. Nielsen, A. Stefani, C. Markos, O. Bang, Temperature insensitive hysteresis free highly sensitive polymer optical fiber Bragg grating humidity sensor, Optics Express 24 (2) (2016) 1206-13.

[14] K. E. Carroll, C. Zhang, D. J. Webb, K. Kalli, A. Argyros, M. C. Large, Thermal response of Bragg gratings in PMMA microstructured optical fibers, Optics Express 15 (14) (2007) 8844-50.

[15] G. Rajan, B. Liu, Y. H. Luo, E. Ambikairajah, G. D. Peng, High Sensitivity Force and Pressure Measurements Using Etched Singlemode Polymer Fiber Bragg Gratings, IEEE Sensors Journal 13 (5) (2013) 17941800 .

[16] G. Rajan, Y. M. Noor, B. Liu, E. Ambikairaja, D. J. Webb, G.-D. Peng, A fast response intrinsic humidity sensor based on an etched singlemode polymer fiber Bragg grating, Sensors and Actuators A: Physical 203 (2013) 107-111.

[17] K. Bhowmik, G. D. Peng, Y. H. Luo, E. Ambikairajah, V. Lovric, W. R. Walsh, G. Rajan, High Intrinsic Sensitivity Etched Polymer Fiber Bragg Grating Pair for Simultaneous Strain and Temperature Measurements, IEEE Sensors Journal 16 (8) (2016) 2453-2459.

[18] D. Saez-Rodriguez, K. Nielsen, H. K. Rasmussen, O. Bang, D. J. Webb, Highly photosensitive polymethyl methacrylate microstructured polymer optical fiber with doped core, Optics Letters 38 (19) (2013) 376972 . 
[19] D. Cohn, A. Hotovely Salomon, Designing biodegradable multiblock PCL/PLA thermoplastic elastomers, Biomaterials 26 (15) (2005) 22972305.

[20] A. Stefani, S. Andresen, W. Yuan, N. Herholdt-Rasmussen, O. Bang, High Sensitivity Polymer Optical Fiber Bragg Grating Based Accelerometer, IEEE Photonics Technology Letters 24 (2012) 763-765.

[21] Y. Termonia, P. Smith, Kinetic-Model for Tensile Deformation of Polymers, Macromolecules 20 (4) (1987) 835-838.

[22] J. De Boer, The influence of van der Waals' forces and primary bonds on binding energy, strength and orientation, with special reference to some artificial resins, Transactions of the Faraday Society 32 (1936) 10-37.

[23] C. A. Buckley, E. P. Lautenschlager, J. L. Gilbert, Deformation Processing of PMMA into High-Strength Fibers, Journal of Applied Polymer Science 44 (8) (1992) 1321-1330.

[24] D. Bosc, C. Toinen, Tensile Mechanical-Properties and Reduced Internal-Stresses of Polymer Optical-Fiber, Polymer Composites 14 (5) (1993) 410-413.

[25] G. Yeh, R. Hosemann, J. Loboda-akovi, H. akovi, Annealing effects of polymers and their underlying molecular mechanisms, Polymer 17 (4) (1976) 309-318.

[26] K. Schmidt-Rohr, A. S. Kulik, H. W. Beckham, A. Ohlemacher, U. Pawelzik, C. Boeffel, H. W. Spiess, Molecular Nature of the BetaRelaxation in Poly(Methyl Methacrylate) Investigated by Multidimensional NMR, Macromolecules 27 (17) (1994) 4733-4745.

[27] E. Muzeau, J. Perez, G. P. Johari, Mechanical Spectrometry of the Beta-Relaxation in Poly(Methyl Methacrylate), Macromolecules 24 (16) (1991) 4713-4723.

[28] S. C. Kuebler, D. J. Schaefer, C. Boeffel, U. Pawelzik, H. W. Spiess, 2D Exchange NMR Investigation of the -Relaxation in Poly(ethyl methacrylate) as Compared to Poly(methyl methacrylate), Macromolecules 30 (21) (1997) 6597-6609. 
[29] J. F. de Deus, G. P. Souza, W. A. Corradini, T. D. Z. Atvars, L. Akcelrud, Relaxations of Poly(methyl methacrylate) Probed by Covalently Attached Anthryl Groups, Macromolecules 37 (18) (2004) 6938-6944.

[30] J. J. Tribone, J. M. O'Reilly, J. Greener, Analysis of enthalpy relaxation in poly(methyl methacrylate): effects of tacticity, deuteration, and thermal history, Macromolecules 19 (6) (1986) 1732-1739.

[31] P. Stajanca, O. Cetinkaya, M. Schukar, P. Mergo, D. J. Webb, K. Krebber, Molecular alignment relaxation in polymer optical fibers for sensing applications, Optical Fiber Technology 28 (2016) 11-17.

[32] K. Fukao, S. Uno, Y. Miyamoto, A. Hoshino, H. Miyaji, Dynamics of and processes in thin polymer films: Poly(vinyl acetate) and poly(methyl methacrylate), Physical Review E 64 (5) (2001) 051807.

[33] S. Saito, T. Nakajima, Glass transition in polymers, Journal of Applied Polymer Science 2 (4) (1959) 93-99.

[34] W. Zhang, D. J. Webb, Factors influencing the temperature sensitivity of PMMA based optical fiber Bragg gratings, in: Micro-Structured and Specialty Optical Fibres III, Vol. 9128, Proc. SPIE, 2014, pp. 91280M91280M.

[35] K. S. Lim, H. Z. Yang, W. Y. Chong, Y. K. Cheong, C. H. Lim, N. M. Ali, H. Ahmad, Axial contraction in etched optical fiber due to internal stress reduction, Optics Express 21 (3) (2013) 2551-62.

[36] P. L. Chu, T. Whitbread, Measurement of stresses in optical fiber and preform, Applied Optics 21 (23) (1982) 4241-4245.

[37] P. Ji, A. D. Q. Li, G.-D. Peng, Transverse birefringence in polymer optical fiber introduced in drawing process, in: International Society for Optics and Photonics, Vol. 5212, Proc. SPIE, 2003, pp. 108-116.

[38] M. K. Szczurowski, T. Martynkien, G. Statkiewicz-Barabach, W. Urbanczyk, L. Khan, D. J. Webb, Measurements of stress-optic coefficient in polymer optical fibers, Optics Letters 35 (2010) 2013-2015.

[39] J. R. Saffell, A. H. Windle, The influence of thermal history on internal stress distributions in sheets of PMMA and polycarbonate, Journal of Applied Polymer Science 25 (6) (1980) 1117-1129. 